\title{
Magnetophotoluminescence of $\mathrm{Zn}_{0.88} \mathrm{Mn}_{0.12}$ Se grown by metal-organic chemical vapor deposition on GaAs substrates
}

\author{
S. L. Lu, ${ }^{\text {a) }}$ D. S. Jiang, ${ }^{\text {b) }}$ J. M. Dai, ${ }^{\text {c) }}$ C. L. Yang, H. T. He, W. K. Ge, and J. N. Wang ${ }^{\text {d) }}$ \\ Physics Department, Hong Kong University of Sciences and Technology, Clear Water Bay, \\ Hong Kong, China \\ K. Chang \\ SKLSM, Institute of Semiconductors, Chinese Academy of Sciences, Beijing 100083, China \\ J. Y. Zhang and D. Z. Shen \\ LESP, Changchun Institute of Optics, Fine Mechanics, and Physics, CAS, Changchun 130033, Jilin, China
}

(Received 21 April 2005; accepted 27 February 2006; published online 11 April 2006)

\begin{abstract}
Magnetophotoluminescence properties of $\mathrm{Zn}_{0.88} \mathrm{Mn}_{0.12} \mathrm{Se}$ thin films grown by metal-organic chemical vapor deposition on GaAs substrates are investigated in fields up to $10 \mathrm{~T}$. The linewidth of the excitonic luminescence peaks decreases with the increasing magnetic field $(<1 \mathrm{~T})$, but the peak energy is almost unchanged. There is a crossover of the photoluminescence intensities between interband and bound excitonic transitions as the magnetic field is increased to about $1 \mathrm{~T}$. These behaviors are interpreted by the strong tuning of the local alloy disorder potential by the applied magnetic field. In addition, the magnetic field-induced suppression of the energy transfers from excitons to $\mathrm{Mn}^{2+}$ ions is also observed. (C) 2006 American Institute of Physics.
\end{abstract}

[DOI: $10.1063 / 1.2190722]$

\section{INTRODUCTION}

$\mathrm{Zn}_{1-x} \mathrm{Mn}_{x} \mathrm{Se}$ belongs to a class of materials called diluted magnetic semiconductors (DMS), where their cations in a nonmagnetic compound are partially replaced by manganese ions. In these materials the $\mathrm{Mn}$ ion is isovalent with the cation. The magnetic properties of these materials are dominated by the $s p-d$ exchange interaction between band electrons and localized $\mathrm{Mn}^{2+}$ ions. ${ }^{1,2}$ During the past few years, extensive investigations have been devoted to the research on $\mathrm{Zn}_{1-x} \mathrm{Mn}_{x} \mathrm{Se}^{3-8}$ The recent interests in spintronics stimulate more attention on this system. ${ }^{9-12}$ Unfortunately, the incorporation of the Mn composition into the alloys induces a larger lattice mismatch, which might generate a huge number of defects and deteriorate the sample quality. Consequently, the optical properties will be greatly affected. In recent years, the material growth technique of $\mathrm{Zn}_{1-x} \mathrm{Mn}_{x} \mathrm{Se}$ has been improved greatly. However, fewer researches related to $\mathrm{Zn}_{1-x} \mathrm{Mn}_{x} \mathrm{Se}$ epilayers grown by metal-organic chemical vapor deposition (MOCVD) are reported compared to researches on the same epilayers grown by molecular beam epitaxy (MBE), although the former is more suitable for large scale production.

In this paper, magnetophotoluminescence (MPL) properties of the $\mathrm{Zn}_{0.88} \mathrm{Mn}_{0.12} \mathrm{Se}$ epilayer grown by MOCVD on GaAs are measured with applied magnetic fields up to $10 \mathrm{~T}$

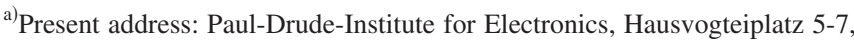
Berlin, Germany.

${ }^{b)}$ Permanent address: SKLSM, Institute of Semiconductors, Chinese Academy of Sciences, Beijing, China.

${ }^{c)}$ Permanent address: Physics Department, Huaibei Coal Industrial Normal College, Huaibei 235000, China.

d) Author to whom correspondence should be addressed; electronic mail: phjwang@ust.hk
}

and at a temperature of $1.6 \mathrm{~K}$. In contrast to the conventional MPL results of DMS, at low magnetic fields $(<1 \mathrm{~T})$ a few interesting features are observed with the increasing magnetic field, including the reduction of the excitonic photoluminescence (PL) peak linewidth, the unchanged peak energy, and the great enhancement of the peak intensity. These results are explained by the strong tuning of the local alloy disorder potential by the applied magnetic field.

\section{EXPERIMENT}

$\mathrm{Zn}_{1-x} \mathrm{Mn}_{x}$ Se epitaxial thin films were grown by MOCVD on (100) GaAs substrates. The Mn compositions $x$ were $5.8 \%, 12 \%$, and $19 \%$. All the samples were grown under similar conditions and with the same thickness of $150 \mathrm{~nm}$. The x-ray diffraction rocking curve and PL spectra of these samples were measured. The high crystalline quality of these samples and the systematic Mn doping effect were confirmed, as has been reported elsewhere. ${ }^{13}$ The MPL measurements were performed in the Faraday geometry at $1.6 \mathrm{~K}$ under a magnetic field varying between 0 and $10 \mathrm{~T}$. The excitation photon energy was either higher $(325 \mathrm{~nm}$ line of a $\mathrm{He}-\mathrm{Cd}$ cw laser) or lower (488 nm line of an $\mathrm{Ar}^{+}$laser) than the band gap energy of $\mathrm{ZnMnSe}$ films. An optical fiber bundle was used to excite the sample.

\section{RESULTS AND DISCUSSIONS}

Here we focus on the sample with the Mn composition of $12 \%$. Figures 1(a) and 1(b) show the dependence of the PL spectra (with $325 \mathrm{~nm}$ laser line excitation) on the magnetic field $B$ of the $\mathrm{Zn}_{0.88} \mathrm{Mn}_{0.12} \mathrm{Se}$ epilayer for the band edge and intra- $\mathrm{Mn}^{2+}$ transitions, respectively. It is found that an efficient transfer of energy between the excitons and $\mathrm{Mn}^{2+}$ ions takes place when the excitation energy of the laser line 

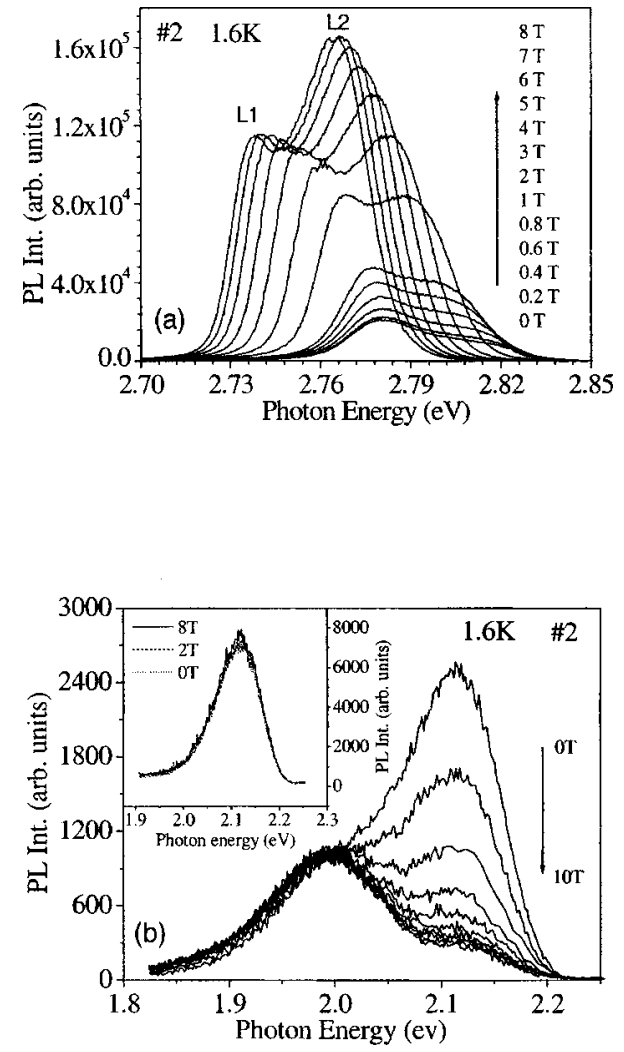

FIG. 1. PL spectra of $\mathrm{Zn}_{0.88} \mathrm{Mn}_{0.12}$ Se obtained at different magnetic fields $B$. (a) The near band edge emission with two excitonic peaks labeled L1 and L2 $(B$ increases with a step of $0.2 \mathrm{~T}$ from 0 to $1 \mathrm{~T}$ and a step of $1 \mathrm{~T}$ from 1 to $8 \mathrm{~T}$ ). (b) The $\mathrm{Mn}^{2+}$ ion and defect-related luminescence with an excitation energy of $3.815 \mathrm{eV}$ higher than energy gap of $\mathrm{ZnMnSe}(B$ increases with a step of $1 \mathrm{~T}$ from 0 to $10 \mathrm{~T}$ ). The inset of (b) shows the PL spectra with an excitation energy of $2.54 \mathrm{eV}$ less than the energy gap of ZnMnSe at the indicated $B$. The arrows indicate the increasing direction of $B$.

is larger than the energy gap of ZnMnSe. As Fig. 1(a) shows, two PL peaks appear in the near band edge region of the spectra, and their intensities are enhanced significantly with the increasing magnetic field. The higher energy peak (labeled L2) has been assigned to the interband (from conduction band to valence band) excitonic transition. The lower one (labeled L1) is assigned to the recombination of the impurity bound exciton, which has been verified through temperature dependent PL and time-resolved PL measurements described in Ref. 13. It is found that the intensity of the impurity bound exciton (peak L1) is relatively stronger in the samples with higher Mn mole fractions, implying that the related bound states result from the Mn incorporation induced potential fluctuation. In fact, it has been shown that in CdMnTe systems, Mn ions taking the substitutional sites of $\mathrm{Cd}$ may cause potential fluctuations in the energy gap and result in severe alloy disorders. ${ }^{14}$ Figure 1(b) depicts the magnetic field-dependent PL spectra in the energy range of $1.8-2.3 \mathrm{eV}$. The peak at $2.1 \mathrm{eV}$ is assigned to the radiative recombination due to the intra- $\mathrm{Mn}^{2+}$ transitions from the ${ }^{4} T_{1(2)}$ excited state $(S=3 / 2)$ to the ${ }^{6} A_{1}(S=5 / 2)$ ground state. Its intensity is greatly reduced with the increasing magnetic field. As a result, the lower energy peak at $2.0 \mathrm{eV}$ becomes more prominent; its emission strength and peak position, however, do not change with the applied magnetic field. This

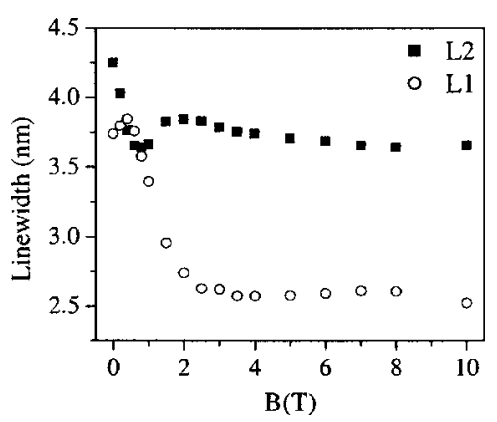

FIG. 2. $B$ dependence of the linewidth of excitonic peaks L2 (solid squares) and $\mathrm{L} 1$ (open circles) in $\mathrm{Zn}_{0.88} \mathrm{Mn}_{0.12} \mathrm{Se}$.

peak is attributed to the luminescence related to selfactivated deep level defects in $\mathrm{Zn}_{0.88} \mathrm{Mn}_{0.12} \mathrm{Se}$, and it has no correlation with Mn ions. The observed PL intensity switching between excitonic emissions (L1 and L2) and the intra$\mathrm{Mn}^{2+}$ emission $(2.1 \mathrm{eV})$ is attributed to the magnetic fieldinduced suppression of the energy transfers from the conduction band electrons to the $\mathrm{Mn}^{2+}{ }^{4} T_{1(2)}$ state, ${ }^{15}$ which is due to spin-dependent selection rules for single ion assisted Augerlike processes. ${ }^{16}$ It is worth noting that when the photon energy of the excitation light is $2.54 \mathrm{eV}(\lambda=488 \mathrm{~nm})$, which is less than the band gap of $\mathrm{Zn}_{0.88} \mathrm{Mn}_{0.12} \mathrm{Se}$ (therefore no electrons are excited from the valence band into the conduction band and the intra- $\mathrm{Mn}^{2+}$ transition is directly excited), the intensity of the intra- $\mathrm{Mn}^{2+}$ luminescence peak shows no significant change with the applied magnetic field, as shown in the inset of Fig. 1(b). This provides a direct evidence that the magnetic field-induced reduction of the luminescence intensity for the intra- $\mathrm{Mn}^{2+}$ peak is related to the energy transfer. Meanwhile, the deep level luminescence $(2.0 \mathrm{eV})$ disappears as the deep levels can no longer trap carriers created by the band gap excitation.

Figure 2 plots the magnetic field dependence of the PL emission linewidth for excitonic peaks L1 and L2 from the above measurements. A striking feature is the decrease of the linewidth with the increasing magnetic field for both peaks $\mathrm{L} 2$ and $\mathrm{L} 1$ in the low field region $(<1 \mathrm{~T})$. The linewidth then becomes almost constant when the magnetic field is beyond $2 \mathrm{~T}$. This linewidth reduction can be explained by the effect of applying a magnetic field on the potential fluctuation induced by an alloy disorder. As proposed by Crooker et al. ${ }^{17}$ the alloy disorder will lead to spatially varying exciton energies near the $\mathrm{Mn}^{2+}$ ions and inhomogeneously broadening PL peak linewidths. The magnetic field will result in a so-called field-induced annealing of the magnetic fluctuation potential based on the assumption that the local conduction and valence band edges near a magnetic $\mathrm{Mn}^{2+}$ cation are closely tied to its spin orientation due to the strong $s p-d$ exchange interaction. It is estimated that in a magnetic field the local band gap near a $\mathrm{Mn}^{2+}$ moment might have a variation in the order of $1 \mathrm{eV}$. Therefore, at low temperatures even a modest field $(<1 \mathrm{~T})$ can dramatically shift the effective energy gap near the $\mathrm{Mn}^{2+}$ cation towards the bulk $\mathrm{ZnSe}$ energy gap, i.e., significantly changing the alloy disorder potential experienced by excitons via aligning the isolated $\mathrm{Mn}^{2+}$ spins. When the magnetic field is strong, the field-induced annealing ef- 


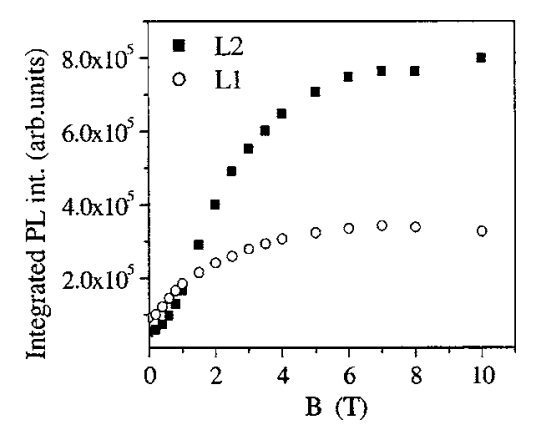

FIG. 3. $B$ dependence of the integrated PL intensity of excitonic peaks L2 (solid squares) and $\mathrm{L} 1$ (open circles) in $\mathrm{Zn}_{0.88} \mathrm{Mn}_{0.12} \mathrm{Se}$.

fect will be saturated, and no further reduction of the linewidth will be achieved. In the case of $\mathrm{Zn}_{1-x} \mathrm{Mn}_{x}$ Se epilayers, the incorporation of $\mathrm{Mn}$ induces such an alloy disorder, and it has been shown from our earlier work $^{13}$ that the rapid thermal annealing effectively increases the composition uniformity and improves the sample quality. Therefore, it is believed that the effect of the applied magnetic field on the alloy disorder strongly affects the linewidth of excitonic luminescence peaks L2 and L1 and is also a kind of annealing process. $^{18}$

Figure 3 shows the integrated PL intensities as a function of the magnetic field for the two excitonic peaks, L2 and L1. The intensity of L2, I(L2), is lower than that of L1, I(L1), at zero magnetic fields. But $I(\mathrm{~L} 2)$ increases much faster than $I(\mathrm{~L} 1)$ as the magnetic field is increased. At about $1 \mathrm{~T}$, there is a crossover, and $I(\mathrm{~L} 2)$ becomes higher than $I(\mathrm{~L} 1)$ afterwards. A similar but larger effect is observed for a sample with a higher Mn composition. With the increasing magnetic field the ratio $I(\mathrm{~L} 2) / I(\mathrm{~L} 1)$ increases. The magnetic field has a strong effect to enhance the relative intensity of peak L2 in comparison with that of peak L1. On the other hand, it is also found that at a zero magnetic field the relative intensity of $I(\mathrm{~L} 2) / I(\mathrm{~L} 1)$ decreases with the increasing Mn composition due to the increasing alloy disorder induced by the $\mathrm{Mn}$ incorporation. ${ }^{13}$ These behaviors can be understood based on the magnetic field-induced competition between the interband and bound excitons. The bound states are induced by a potential disturbance due to the $\mathrm{Mn}$ incorporation and are therefore related to the alloy disorder in the $\mathrm{Zn}_{0.88} \mathrm{Mn}_{0.12} \mathrm{Se}$. As a result, the field-induced "annealing" of the alloy potential fluctuations leads to a fast saturation of $I(\mathrm{~L} 1)$ as the magnetic field increases. On the other hand, I(L2) continues to increase with the increasing magnetic field. The magnetic field acts as an additional confining potential which squeezes the excitons, enhances the electron-hole pair recombination probability, ${ }^{19-21}$ and, in addition, suppresses the energy transfers to $\mathrm{Mn}^{2+}$ ions. ${ }^{15}$ Therefore, the crossover occurs, and $I(\mathrm{~L} 2)$ becomes higher than $I(\mathrm{~L} 1)$ at a certain magnetic field. This is consistent with the behavior of the linewidth variation; i.e., both are due to the field-induced annealing of the alloy potential fluctuations.

Figure 4 shows the dependence of the magnetic fieldinduced peak energy shift $\Delta E[=E(B)-E(0)]$ for the interband exciton PL peak L2 on the applied magnetic field. The solid squares are the experimental data. At low magnetic

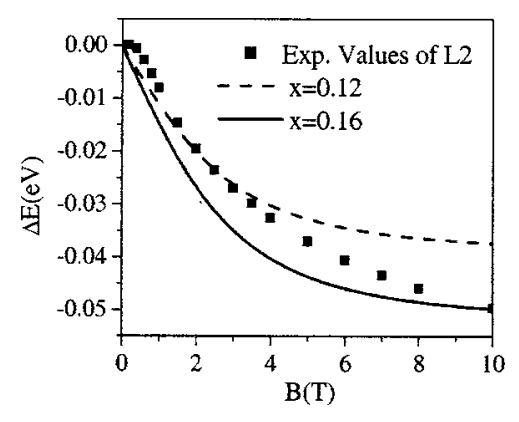

FIG. 4. $B$ dependence of PL peak energies for L2 (solid squares) in $\mathrm{Zn}_{0.88} \mathrm{Mn}_{0.12} \mathrm{Se}$. The theoretical calculated dependence is shown by a dashed line (Mn composition $x=0.12)$ and a solid line $(x=0.16)$.

fields $(<1 \mathrm{~T})$, the PL peak position of L2 is weakly affected by the magnetic field. But a large red shift of the PL peak is observed at higher magnetic fields. A similar behavior is observed for the PL emission peak L1. The weak effect of the magnetic field on the PL peak position at low fields $(<1 \mathrm{~T})$ might be due to the fact that in our experiment, the $\sigma^{+}$and $\sigma^{-}$circular polarization were not distinguished during the measurement. Due to the Zeeman effect the band edge $\sigma^{+}$ transition energy decreases with the increasing magnetic field, while the $\sigma^{-}$transition energy increases with the increasing magnetic field. At low magnetic fields, the reduction of the alloy disorder induced inhomogeneous broadening of transition energies and the combined PL signal from both polarizations lead to an apparent weak $B$ dependence of the exciton peak. At high $B$, however, the energy splitting between $\sigma^{+}$and $\sigma^{-}$transitions is larger and the reduction of the alloy disorder induced inhomogeneous broadening is saturated; the lower luminescence peak from the $\sigma^{+}$transition is enhanced and becomes dominant due to the Boltzmann distribution of the carriers. Therefore, the peak position starts to decrease rapidly with the increasing $B$ as observed in our experimental results.

The solid and dashed lines in Fig. 4 are the calculated results for $\mathrm{Zn}_{1-x} \mathrm{Mn}_{x} \mathrm{Se}$, with $x=0.16$ and 0.12 , respectively, based on the magnetic field- induced energy shift of luminescence transitions with the $\sigma^{+}$polarization. It is noted that the energy shift $\Delta E$ of the peak position is large due to the $s p$ - $d$ exchange interaction between the carriers and the magnetic ions, and is dependent on the Mn composition $x$. An important feature of the $s p-d$ interaction is the giant Zeeman effect. The giant Zeeman shift $E_{z}$ is given by

$$
E_{Z}=(\alpha-\beta) N_{0} x S_{0} B_{5 / 2}\left\{\frac{5 m_{B} g_{\mathrm{Mn}} B}{2 k\left(T+T_{\mathrm{AF}}\right)}\right\},
$$

where $x$ is the Mn concentration, $B_{5 / 2}$ is a Brillouin function, $N_{0}$ is the number of unit cells per unit volume, and $N_{0} \alpha$ $=260 \mathrm{meV}$ and $N_{0} \beta=-1310 \mathrm{meV}$ are the exchange energies for electrons and holes, respectively. The phenomenological parameters $S_{0}=0.41$ and $T_{\mathrm{AF}}=3.6 \mathrm{~K}$ take into account the antiferromagnetic coupling between neighboring $\mathrm{Mn}^{2+}$ ions. ${ }^{8,22}$ Comparing the calculated and measured results, the measured large red shift of the peak energy is qualitatively consistent with the theory at higher magnetic fields. The deviation from the calculated curves for fixed Mn compositions is possibly caused by the alloy disorder in $\mathrm{ZnMnSe}$. 


\section{CONCLUSIONS}

In conclusion, for the $\mathrm{Zn}_{0.88} \mathrm{Mn}_{0.12}$ Se epilayer grown by MOCVD on GaAs, the excitonic PL linewidth reduction and the weak dependence of PL peak energies at low magnetic fields $(<1 \mathrm{~T})$ can be well understood by the magnetic fieldinduced "annealing" of the alloy potential fluctuations originating from the Mn-induced disorder. The observed quenching of the $\mathrm{Mn}^{2+}$ intraion transition with the increasing magnetic field results from the magnetic field-induced suppression of the energy transfer from excitons to $\mathrm{Mn}^{2+}$ ions.

\section{ACKNOWLEDGMENT}

This work is supported by the UGC of Hong Kong SAR via DAG03/04.SC26 and HIA03/04/SC02.

${ }^{1}$ J. K. Furdyna, J. Appl. Phys. 64, R29 (1988).

${ }^{2}$ A. Twardowski, T. Dietl, and M. Demianiuk, Solid State Commun. 48, 845 (1983).

${ }^{3}$ R. B. Bylsma, W. M. Becker, J. Kossut, and U. Debska, Phys. Rev. B 33, 8207 (1986).

${ }^{4}$ A. K. Aora, E. K. Suh, U. Debska, and A. K. Ramdas, Phys. Rev. B 37, 2927 (1988).

${ }^{5}$ N. Dai, H. Luo, F. C. Zhang, N. Samarth, M. Dobrowolska, and J. K. Furdyna, Phys. Rev. Lett. 67, 3824 (1991).

${ }^{6}$ W. C. Chou et al., J. Appl. Phys. 75, 2936 (1994).

${ }^{7}$ C. T. Tsai, D. S. Chuu, J. Y. Leou, and W. C. Chou, Jpn. J. Appl. Phys., Part 1 36, 4427 (1997).
${ }^{8}$ P. J. Klar, D. Wolverson, J. J. Davies, W. Heimbrodt, and M. Happ, Phys. Rev. B 57, 7103 (1998).

${ }^{9}$ B. T. Jonker, Y. D. Park, B. R. Bennett, H. D. Cheong, C. Kioseoglou, and A. Petrou, Phys. Rev. B 62, 8180 (2000).

${ }^{10}$ E. Oh et al., Appl. Phys. Lett. 83, 4604 (2003).

${ }^{11}$ D. Litvinov, D. Gerthsen, A. Rosenauer, B. Daniel, and M. Hetterich, Appl. Phys. Lett. 85, 751 (2004).

${ }^{12}$ W. M. Chen et al., Appl. Phys. Lett. 85, 5260 (2005).

${ }^{13}$ S. L. Lu et al., J. Cryst. Growth 249, 538 (2003).

${ }^{14}$ A. Golnik, J. Ginter, and J. A. Gaj, J. Phys. C 16, 6073 (1983).

${ }^{15}$ C. S. Kim, M. Kim, S. Lee, J. Kossut, J. K. Furdyna, and M. Dobrowolska, J. Cryst. Growth 214/215, 395 (2000).

${ }^{16}$ M. Nawrocki, Yu. G. Rubo, J. P. Lascaray, and D. Coquillat, Phys. Rev. B 52, R2241 (1995).

${ }^{17}$ S. A. Crooker, D. G. Rickel, S. K. Lyo, N. Samarth, and D. D. Awschalow, Phys. Rev. B 60, R2173 (1999).

${ }^{18}$ Increasing magnetic fields in the $z$ direction will squeeze exciton orbits in the $x-y$ plane, and the effective sweeping area will be reduced. This reduced sweeping area may lead to a reduction in LW if it is comparable to the average lateral extension of disorder in the $x-y$ plane. In our sample, we believe that the average lateral extension of disorder is much smaller than the excitonic orbits. The reduced sweeping area by increasing $B$ will not result in a significant LW reduction. Otherwise, we would expect to see a continuous reduction in LW with increasing $B$. But this is not what we have observed.

${ }^{19}$ A. Polimeni, S. T. Stoddart, M. Henini, L. Eaves, P. C. Main, K. Uchida, R. K. Hayden, and N. Miura, Physica E (Amsterdam) 2, 662 (1998).

${ }^{20}$ V. Halonen, T. Chakraborty, and P. Pietiläinen, Phys. Rev. B 45, 5980 (1992).

${ }^{21}$ S. Jaziri and R. Bennaceur, Semicond. Sci. Technol. 9, 1775 (1994).

${ }^{22}$ J. M. Fatah, T. Piorek, P. Harrison, and W. E. Hagstron, Phys. Rev. B 49, 10341 (1994). 